\title{
Performance Implications of Three-Mirror Fabry-Perot Demultiplexers for 10-Gb/s WDM Dispersion-Supported Transmission with 0.5-nm Channel Spacing
}

\author{
Mário M. Freire, Álvaro M. F. de Carvalho, and Henrique J. A. da Silva
}

\begin{abstract}
This letter assesses the performance of dispersionsupported transmission (DST) for three 10-Gb/s WDM channels separated $0.5 \mathrm{~nm}$, using a three-mirror Fabry-Perot demultiplexer. It is shown that the use of three-mirror demultiplexers reduces the crosstalk penalty to less than $1 \mathrm{~dB}$ in the region of small linear increase of dispersion penalty of the DST method, while double-cavity Fabry-Perot demultiplexers are less suitable to operate at this channel spacing. Compared with published performance studies for WDM-DST systems with $1 \mathrm{~nm}$ of channel spacing, these results indicate the channel spacing may be cut in a half, if a three-mirror filter is used as demultiplexer.
\end{abstract}

\section{INTRODUCTION}

I F $10 \mathrm{~Gb} / \mathrm{s}$ wavelength division multiplexed (WDM) channels are to be transmitted via long spans of standard singlemode fibers (SMF) using the method of dispersion-supported transmission (DST) [1], narrow-channel spacings are required in order to reduce the differences on the system performance due to the EDFA gain dependence with wavelength, and due to a different value of fiber dispersion at the wavelength assigned to each channel. Using WDM and polarizationdivision multiplexing (PDM), four 10-Gb/s channels separated $0.5 \mathrm{~nm}$ have been transmitted over $340 \mathrm{~km}$ of dispersion shifted fiber (DSF) [2]. Using three tunable bandpass filters in series for channel selection, seventeen $20 \mathrm{~Gb} / \mathrm{s}$ WDM channels have been transmitted through $150 \mathrm{~km}$ of dispersion-managed fiber (DMF), with 0.8-nm channel spacing which corresponds to 5 times the bit rate [3].

Performance assessment of multichannel dispersionsupported transmission using single- and double-cavity Fabry-Perot (FP) demultiplexers has been reported for two [4] and three [5] $10 \mathrm{~Gb} / \mathrm{s}$ WDM channels separated $1 \mathrm{~nm}$.

In this letter, a system performance comparison is presented for three-mirror and double-cavity Fabry-Perot demultiplexers.

Manuscript received March 21, 1996; revised May 13, 1996

M. M. Freire is with the Department of Mathematics and Computer Science, University of Beira Interior, Rua Marquês d'Ávila e Bolama, 6200 Covilhã, Portugal.

Á. M. F. de Carvalho and H. J. A. da Silva are with the Department of Electrical Engineering, University of Coimbra, Largo Marquês de Pombal, 3030 Coimbra, Portugal.

Publisher Item Identifier S 1041-1135(96)06567-6.

\section{FREQUENCY RESPONSE}

In this section, we compare the frequency response of double-cavity and three-mirror FP filters. The frequency response of a double-cavity FP filter, with equal cavities, is given by the product of two field transfer functions of singlecavity FP filters [6]. Neglecting losses, the full width at half maximum (FWHM) of the frequency response for a doublecavity FP filter (DC-FPF), with equal cavities, can be shown to be

$$
\mathrm{FWHM}=\sqrt{\sqrt{2}-1} \frac{\mathrm{FSR}}{F}
$$

where FSR is the free spectral range of each cavity, and $F$ is the finesse of each cavity. The three-mirror FP filter is assumed to be symmetric with identical outer mirrors, as well as identical lengths. For this conditions, and neglecting losses, the frequency response of a three-mirror FP filter is [7]

$$
H_{\mathrm{TMF}}(\Omega)=\frac{\sqrt{T_{0}} T_{1}}{1-2 \sqrt{R_{0} R_{1}} e^{-(j \Omega / \mathrm{FSR})}+R_{1} e^{-(2 j \Omega / \mathrm{FSR})}}
$$

where $R_{0}$ and $R_{1}$ are the reflectances of the center and outer mirrors, respectively, $T_{0} e T_{1}$ are the corresponding transmittances, and FSR is the free spectral range of each half-cavity. The frequency response of this filter is of the second-order Butterworth type, if $R_{0}$ is chosen to have the critical value:

$$
R_{0}=\frac{4 R_{1}}{\left(1+R_{1}\right)^{2}} .
$$

Details about the measured shape of the bandpass of a threemirror FPF, with the reflectance of the center mirror slightly lower than its critical value and with an insertion loss of 4.5 $\mathrm{dB}$, can be found in [7]. After some algebraic manipulations, it can be shown that the full width at half maximum (FWHM) of the frequency response for this filter is given by

$$
\mathrm{FWHM}=\frac{\mathrm{FSR}}{\pi} \operatorname{arc} \cos \left(1-\sqrt{1+\frac{\beta}{4 R_{1}}}\right)
$$

with

$$
\beta=2 T_{0} T_{1}^{2}+R_{1}\left(2-R_{1}-4 R_{0}\right)-1 .
$$

Fig. 1 shows the power transfer function of a double-cavity and a three-mirror FP filter, both with a FWHM of $50 \mathrm{GHz}$. 


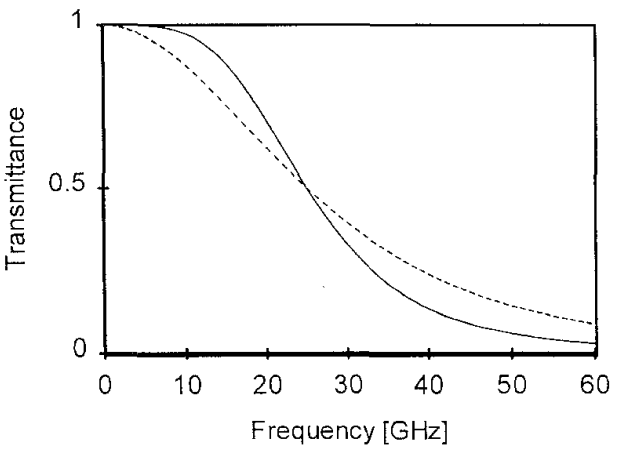

Fig. 1. Comparison of the power transfer function for a three-mirror (TM) FPF and a double-cavity ( - - DC) FPF, both with FWHM $=50 \mathrm{GHz}$.

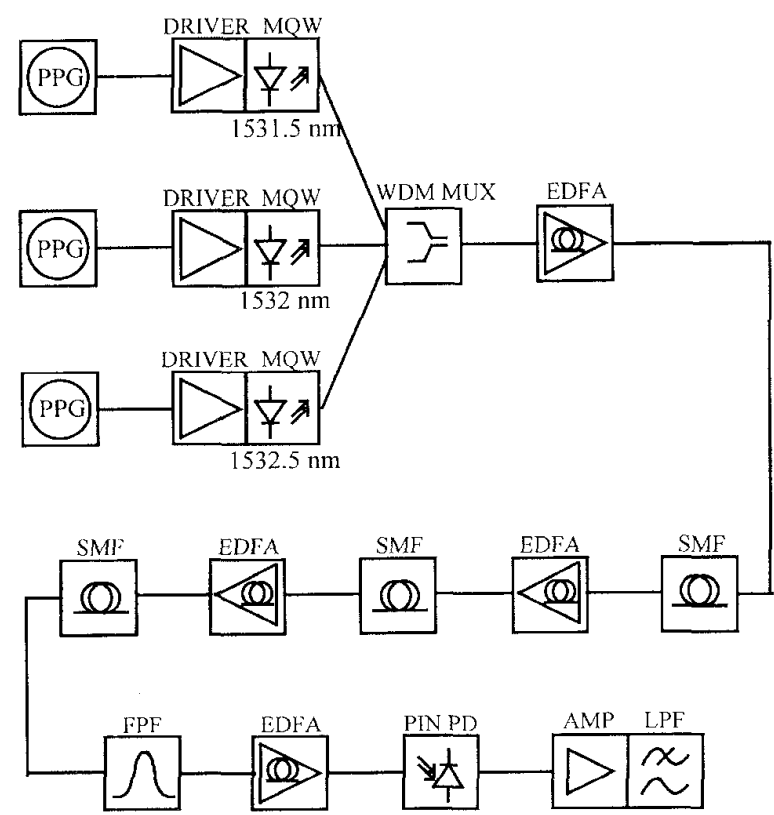

Fig. 2. Block diagram of the simulated WDM-DST system.

As can be seen, the double-cavity filter has a frequency response which is the product of two approximately Lorentzian responses, whereas the three-mirror filter has a frequency response of second-order Butterworth type.

Other (de)multiplexer technologies that can be modeled as a third-order Butterworth filter [8], may permit an increase in the channel density, but they are a subject for futher study.

\section{Modeling and Performance Evaluation}

The block diagram of the simulated WDM-DST system is shown in Fig. 2. The system model is similar to the ones reported in [4], [5]. A pseudorandom binary sequence (PRBS) of $2^{7}-1$ bits has been used to assess the performance of WDM systems [4], [5], [8]. Since the three-mirror FPF is a linear sub-system, a PRBS with $2^{7}-1$ bits at $10-\mathrm{Gb} / \mathrm{s}$ is also assumed to be provided by each pseudopattern generator (PPG). Each optical transmitters consists of a laser driver, and a MQW-DFB laser. The dynamic response of each MQWDFB laser has been described by a rate equation model, which takes into account the carrier transport effects. The values of

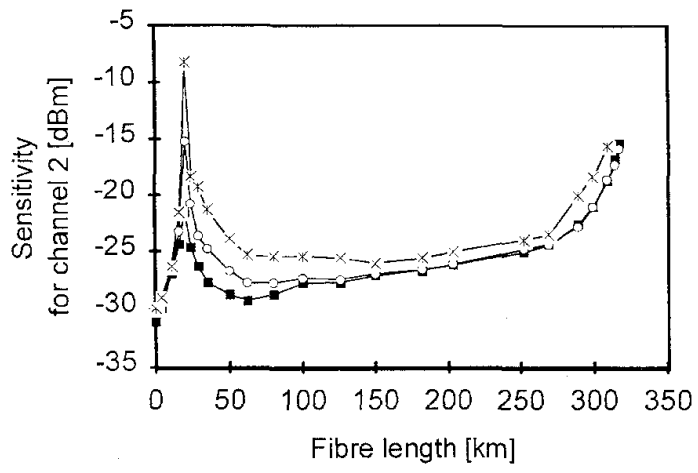

Fig. 3. Receiver sensitivity for channel 2 versus fiber length, assuming that a double-cavity (DC) or a three-mirror (TM) FP demultiplexer is used. For comparison, the receiver sensitivity for single-channel DST is also displayed. $-\star-\mathrm{DC}(\mathrm{ch} .1+\mathrm{ch} .2+\mathrm{ch} .3) .-\mathrm{o}-\mathrm{TM}(\mathrm{ch} .1+\mathrm{ch} .2+\mathrm{ch} .3)$. DST (ch.2).

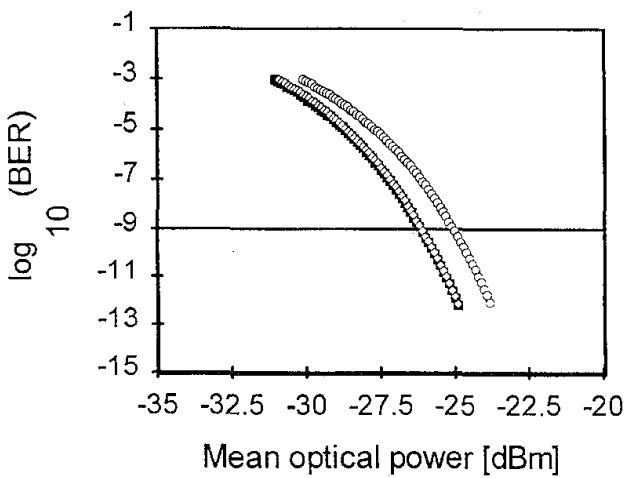

Fig. 4. Average error probability for channel 2 versus mean optical power at the input of the optical preamplifier, after transmission via $204 \mathrm{~km}$ SMF. A double-cavity (DC) or a three-mirror (TM) FPF is assumed to be used as demultiplexer. For comparison, the average error probability for single-channel DST is also displayed. $-0-D C(\mathrm{ch} .1+\mathrm{ch} .2+\mathrm{ch} .3) .-\diamond-$ $\mathrm{TM}(\mathrm{ch} .1+\mathrm{ch} .2+$ ch. 3$) . \longrightarrow \mathrm{DST}(\mathrm{ch} .2)$

the laser parameters used in the simulations were obtained from [9] and the emission wavelengths of the lasers were $1531.5 \mathrm{~nm}$ (channel 1), $1532 \mathrm{~nm}$ (channel 2) and $1532.5 \mathrm{~nm}$ (channel 3). At the WDM optical multiplexer output, the total electric field is the sum of the input electric fields. The optical amplifiers (EDFA's) have been considered as linear with a noise equivalent bandwidth of $1.25 \mathrm{THz}$ and a spontaneous emission factor $\left(n_{s p}\right)$ of $0.3 \mathrm{~dB}$. They are assumed to be used in the configurations of booster, in-line, and preamplifier, as in the DST experiments [1]. The standard single-mode fiber (SMF) was modeled using the lowpass transfer function with first-order dispersion of $16.2 \mathrm{ps} /(\mathrm{nm} . \mathrm{km})$ at $1532 \mathrm{~nm}$. A threemirror Fabry-Perot filter (TM-FPF) [5] is assumed to be used to select channel 2 (signal channel). The reflectivities of the outer mirrors were considered to be 0.8 and the reflectivity of the center mirror was chosen so that the frequency response of the filter is of the second-order Butterworth type. In order to compare the system performance using a TM-FPF with the performance of WDM-DST systems using a double-cavity FPF (DC-FPF), a DC-FPF with a finesse of each cavity equal to 150 is also considered, as in [4]. Due to imperfect response of the optical demultiplexer, a small portion of the optical power from rejected channels (ch.1 and ch.3) is transferred to the 


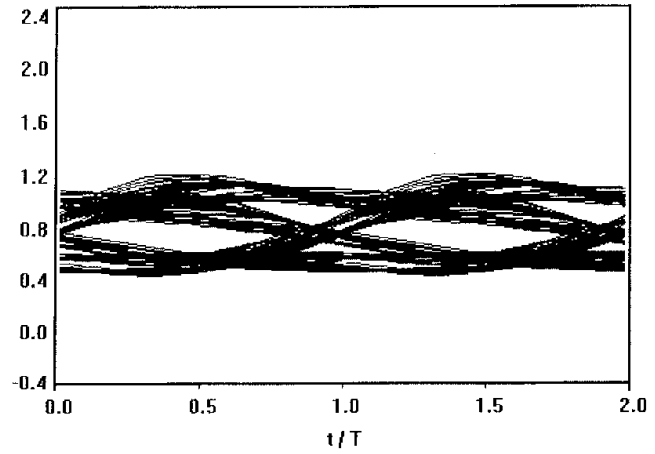

Fig. 5. Eye-diagram at the lowpass filter output after DST via $204 \mathrm{~km} \mathrm{SMF}$ of three $10 \mathrm{~Gb} / \mathrm{s}$ WDM channels separated $0.5 \mathrm{~nm}$ and selection of channel 2 with a TM-FP demultiplexer (FWHM $=30 \mathrm{GHz}$ ).

signal channel resulting in linear crosstalk. A PIN photodiode, with a 3-dB cutoff frequency of $9.35 \mathrm{GHz}$, is assumed to be used. The receiver main amplifier and the lowpass filter (LPF) have been jointly modeled as a lowpass RC filter with the 3-dB bandwidth required by the DST method.

The receiver noise sources include signal-ASE, ASE-ASE, and crosstalk-ASE beat noises, and the average error probability was estimated using the semi-analytical method with Gaussian approximation, as in [4].

\section{SySTEM PERFORMANCE IMPLICATIONS}

The performance assessment was focused on channel 2 . For each fiber length the system parameters, namely the bias current, the modulation current, the FWHM bandwidth of the FPF, and the receiver cutoff frequency, have been adjusted in order to minimize the EDFA preamplifier input mean optical power for an average error probability (BER) of $10^{-9}$.

Fig. 3 shows the receiver sensitivity for channel 2 after three-channel transmission via different fiber lengths, assuming that a three-mirror (TM) or a double-cavity (DC) FPF is used as demultiplexer. For performance comparison, the receiver sensitivity for single-channel DST is also shown. As can be seen, if a three-mirror FP demultiplexer is used the crosstalk penalty is less than $1 \mathrm{~dB}$, in the region of small linear increase of dispersion penalty of the DST method $(80-270 \mathrm{~km})$, and for distances ranging from 100 to $315 \mathrm{~km}$ the crosstalk penalty is less than $0.5 \mathrm{~dB}$. The reduction of crosstalk penalties for these link lengths follows the narrowing of the laser spectra: the laser frequency deviation of $15 \mathrm{GHz}$ at $80 \mathrm{~km}$ rapidly decreases with fiber length, being of 4.8 $\mathrm{GHz}$ at $270 \mathrm{~km}$. If a double-cavity FP demultiplexer is used as demultiplexer, the crosstalk penalty is greater than $1 \mathrm{~dB}$ in the region of small linear increase of dispersion penalty of the DST method $(80-270 \mathrm{~km})$, except around $270 \mathrm{~km}$, where the crosstalk penalty is about $0.9 \mathrm{~dB}$.

The average error probability against mean optical power at the input of the optical preamplifier is displayed in Fig. 4, for single-channel DST and for three-channel DST over $204 \mathrm{~km}$ SMF. As can be seen in this figure, the mean optical power required to achieve a BER of $10^{-9}$ is $-26.17 \mathrm{dBm}$ for single- channel DST, and -26.13 and $-25.04 \mathrm{dBm}$ for three-channel DST with three-mirror (TM) and double-cavity (DC) FP demultiplexers, respectively. Therefore, the crosstalk penalty, at a BER of $10^{-9}$, is less than $0.1 \mathrm{~dB}$ for the three-mirror demultiplexer, whereas for the double-cavity demultiplexer, the crosstalk penalty is greater than $1 \mathrm{~dB}$. Fig. 5 shows the eye-diagram at the lowpass filter output after DST via 204 $\mathrm{km}$ SMF of three $10 \mathrm{~Gb} / \mathrm{s}$ WDM channels separated $0.5 \mathrm{~nm}$ and selection of channel 2 using a TM demultiplexer with a FWHM of $30 \mathrm{GHz}$. This eye-diagram is very similar to the one reported by Wedding et al. for single-channel DST over the same distance [1].

\section{CONCLUSION}

Simulation results show that is possible to transmit three $10 \mathrm{~Gb} / \mathrm{s}$ WDM channels separated $0.5 \mathrm{~nm}$ in the region of small linear increase of dispersion penalty of the DST method $(80-270 \mathrm{~km})$, if a three-mirror Fabry-Perot demultiplexer is used. It was also shown that double-cavity Fabry-Perot demultiplexers are less suitable to operate at this channel spacing. Compared with published performance studies for WDM-DST systems with 1-nm channel spacing, the use of three-mirror demultiplexers allows an increase by a factor of two in the frequency utilization efficiency with low-crosstalk penalties. However, the reduction of channel spacing to $0.5 \mathrm{~nm}$ makes WDM-DST systems more susceptible to laser/demultiplexer misalignments arising from device imperfections, temperature variations and aging.

\section{REFERENCES}

[1] B. Wedding, B. Franz, and B. Junginger, "10-Gb/s optical transmission up to $253 \mathrm{~km}$ Via standard single-mode fiber using the method of dispersion-supported transmission," J. Lightwave Technol., vol. 12, no. 10, pp. $1720-1727,1994$.

[2] K. Sekine, S. Sasaki, and N. Kikuchi, "10 Gbit/s four-channel wavelength- and polarization-division multiplexing transmission over $340 \mathrm{~km}$ with $0.5 \mathrm{~nm}$ channel spacing," Electron. Lett., vol. 31, no. 1, pp. $49-50,1995$.

[3] A. R. Chraplyvy, A. H. Gnauck, R. W. Tkach, R. M. Derosier, C. R. Giles, B. M. Nyman, G. A. Ferguson, J. W. Sulhoff, and J. L. Zyskind, "One-third terabit/s transmission through $150 \mathrm{~km}$ of dispersion-managed fiber," IEEE Photon. Technol. Lett., vol. 7, no. 1, pp. 98-100, 1995.

[4] M. M. Freire and H. J. A. da Silva, "Performance assessment of two-channel dispersion-supported transmission systems using single and double-cavity Fabry-Perot filters as demultiplexers," IEEE Photon. Technol. Lett., vol. 7, no. 11, pp. 1360-1362, 1995.

[5] _ "Performance assessment of WDM dispersion supported transmission systems using single and double-cavity Fabry-Perot demultiplexers," in Proc. LEOS'95, San Francisco, CA, vol. 2, 1995, pp. OC 6.4 .

[6] P. A. Humblet and W. M. Hamdy, "Crosstalk analysis and filter optimization of single- and double-cavity Fabry-Perot filters," IEEE J. Select. Areas Commun., vol. 8, no. 6, pp. 1095-1107, 1990.

[7] J. Stone, L. W. Stulz, A. A. M. Saleh, "Tree-mirror fiber Fabry-Perot filters of optimal design," Electron. Lett., vol. 26, no. 14, pp. 1073-1074, 1990.

[8] N. N. Khrais, A. F. Elrefaie, R. E. Wagner, and S. Ahmed, "Performance degradation of multiwavelength optical networks due to laser and (de)multiplexer misalignments," IEEE Photon. Technol. Lett., vol. 7 , no. 11 , pp. $1348-1350,1995$.

[9] R. F. S. Ribeiro, J. R. F. da Rocha, A. V. T. Cartaxo, H. J. A. da Silva, B. Franz, and B. Wedding, "FM response of quantum-well lasers taking into account carrier transport effects," IEEE Photon. Technol. Lett., vol. 7, no. 8, pp. 857-859, 1995. 